\title{
Editor's Message to Special Issue of Ubiquitous Computing Systems (VII)
}

\author{
Tsutomu Terada ${ }^{1, a)}$
}

In initial stage of ubiquitous computing research, ubiquitous computing means that computers exist everywhere and they provide various services to support human daily life. As a result of the miniaturization/generalization of computers, the research area is extending to adjacent topics including application technologies, communication technologies, and psychological interaction technologies.

This special issue had 29 submissions, and 12 papers were accepted ( $41 \%$ acceptance rate). While the acceptance rate was a bit below 50\% that we expected, we accepted enough number of excellent papers. The topics of accepted papers include power management, network simulation, mobile sensing, social media, position estimation, and context recognition. These topics seemingly have little relationship. However, from the viewpoint of ubiquitous computing system, these papers covers important elements of ubiquitous system. For several years, this special issue was published once a year. You can use this issue as an opportunity to grasp the ubiquitous computing research, and we are very appreciated if you will submit your paper to this special issue.

Finally, I would like to express our sincere appreciation to editorial board members, reviewers, and all authors for their contribution to this special issue.

\section{The Editorial Committee}

\section{- Editor in-Chief:}

Tsutomu Terada (Kobe University).

\section{- Editorial Board:}

Ren Omura (Toyohashi University of Technology)

Yasue Kishino (NTT Corporation)

- Editorial Committee:

Kaori Fujinami (Tokyo University Agriculture and Technology)

Kazuya Murao (Ritsumeikan University)

Takuro Yonezawa (Keio University)

Ismail Arai (Nara Institute of Science and Technology)

Sozo Inoue (Kyushu Institute of Technology)

Yu Enokibori (Nagoya University)

Kazushige Ouchi (TOSHIBA Corporation)

Yasushi Ogawa (Ricoh Corporation)
Arisa Kadomura (Panasonic Corporation) Yoshihiro Kawahara (The University of Tokyo) Quan Kong (Hitachi Ltd.)

Arei Kobayashi (KDDI)

Masamichi Shimosaka (Tokyo Institute of Technology)

Midori Sugaya (Shibaura Institute of Technology)

Yasuyuki Sumi (Future University Hakodate)

Hideyuki Takahashi (Tohoku University)

Emi Tamaki (Waseda University)

Yusuke Doi (Preferred Networks, Inc.)

Jin Nakazawa (Keio University)

Haruo Noma (Ritsumeikan University)

Kei Hiroi (Nagoya University)

Takuya Maekawa (Osaka University)

Kohei Matsumura (Ritsumeikan University)

Akihiro Miyata (Nihon University)

Keiichi Yasumoto (Nara Institute of Science and Technology)

Koji Yatani (The University of Tokyo)

Yutaka Yanagisawa (m plus plus Corporation, Ltd.)

Kazunori Yamada (Panasonic Corporation)

Hiroki Watanabe (Hokkaido University)

Hiroyuki Manabe (NTT DOCOMO, Inc.)

\footnotetext{
Graduate School of Engineering, Kobe University
}

a) tsutomu@eedept.kobe-u.ac.jp 